\title{
XXIV. Der Kupferkies von Pulacayo.
}

\author{
Von \\ Zoltán Toborffy in Budapest.
}

(Hierzu Taf. X.)

Die mineralogische Abtheilung des ungarischen Nationalmuseums bekam neuerdings aus der Grube Pulacayo (in Bolivien) vortieffliche Kupferkiese, mit deren krystallographischer Bearbeitung Prof. Dr. Josef Krenner mich betraute.

Die Resultate meiner Untersuchungen fasse ich im Folgenden zusammen.

Dieser Kupferkies wurde bis jetzt nur durch G. vom Rath 1) erwähnt (1886) in der Beschreibung des Pulacayoer Tetraëdrits, und zwar mit den Worten: „Das Fahlerz wird von ziemlich unscheinbaren Krystallen von brauner Blende und sehr zierlichen Kupferkieskryställchen begleitet. Letztere bilden Zwillinge nach dem Gesetze: Zwillingsebene ist eine Fläche der Grundform. «

An dem untersuchten Materiale konnte ich folgende Formen feststellen: die Sphenoide:

$$
\begin{aligned}
p & =x\{1 \mid 1\} \\
p^{\prime}=r & =x\{1 \mathrm{1} 1\} \\
{ }^{*} x & =x\{113\} \\
x^{\prime} & =x\{1 T 3\} \\
{ }^{*} \eta & =x\{771\}
\end{aligned}
$$

die Pyramiden zweiter Art:

$\begin{array}{lr}e\{101\} & h\{302\} \\ z\{201\} & J\{503\} \\ x\{403\} & g\{203\} \\ \varepsilon\{705\} & * \tau\{509\} \\ \delta\{10.0 .7\} & \Theta\{405\},\end{array}$

1) VerL. der niederrb. Ges. Bonn 1886, 192. 
die Prismen erster und zweiter Art:

$$
m\{110\}, a\{100\},
$$

die Endfläche $e\{001\}$ und das Skalenoëder $\left.y\{313\}^{1}\right)$.

Das Sphenoid $p=x\{111\}$ ist gewöhnlich sehr gerieft, bei einfachen Krystallen nur horizontal, während bei Zwillingen auch eine den Combinationskanten [111:101] und [1T1:101] parallele Riefung hinzutritt, was triangulare Zeichnungen verursacht.

Das negative Sphenoid $p^{\prime}=x\{1 T 1\}$ hat immer glänzende, fehlerlose Flächen und ist, wenn es mit dem positiven zugleich auftritt, immer kleiner als jenes.

$x^{\prime}=x\{1 T 3\}$ stumpft die Combinationskanten von $x\{1 T 1\}$ und $\{001\}$ zonal ab und hat mit $x\{1 T 1\}$ übereinstimmende Eigenschaften.

Das positive Sphenoid $x=x\{113\}$, das ich auch mit dem entsprechenden $x\{1 T 3\}$ zugleich beobachtet hatte, erscheint in gerieften, schmalen Streifen.

Das Sphenoid $\eta=\varkappa\{\mathbf{7 7 1}\}$ konnte nur in einem einzigen Falle bestimmt werden, mit einer zwar gestreiften, aber ziemlich gut reflectirenden Fläche.

Die gewöhnlichsten und fast immer vorhandenen Pyramiden zweiter $\Lambda$ rt sind $e\{101\}, \approx\{201\}$ und $g\{203\}$. Sie sind alle glänzende, schön spiegelnde Flächen; an Ausdehnung pflegt $\{101\}$ die beiden anderen manchmal zu übertreffen. Die übrigen erwähnten Pyramiden erscheinen nur untergeordnet, in schmalen Streifen, zuweilen mit verzerrten, undeutlichen Reflexen.

Die Endfläche $o\{001\}$ ist bald ziemlich gross, bald wieder kaum bemerkbar, glänzend oder gekörnt. Bei der Entzifferung der Zwillingsbildungen leistet sie ihrer vortrefflichen Ausbildung wegen sehr gute Dienste. Horizontal gerieft erscheint $m\{110\}$ entweder nur als abstumpfender Streifen bei den pyramidalen Krystallen, oder als herrschende Form bei dem prismatischen Typus.

Das Prisma zweiter Art a $\{100\}$ stimmt in Bezug auf den Grad des Glanzes mit der Endfläche $o\{001\}$ überein.

Das einzige Skalenoëder $y=x\{113\}$ wurde in zwei Fällen in Form kleiner, jedoch glänzender Flächen beobachtet.

Bemerkenswerth ist an den Krystallen dieses Kupferkieses die bei Erzen seltene Constanz der Flächenwinkel, wie es die folgenden Werthe beweisen.

\begin{tabular}{|c|c|c|}
\hline \multicolumn{2}{|c|}{ Berechnel } & Beobachte \\
\hline$(001):(111)$ & $=54^{\circ} 20^{\prime}$ & $54^{0} 018$ \\
\hline$(001):(101)$ & 44341 & 4435 \\
\hline$(001):(201)$ & $63 \quad 5 \frac{1}{2}$ & $63 \quad 7$ \\
\hline
\end{tabular}

1) Die mit * bezeichneten sind neue Formen. 
Zoltán Toborffy.

\begin{tabular}{|c|c|c|c|}
\hline$(001):(203)=$ & Berechnet: & \multicolumn{2}{|c|}{$\begin{array}{c}\text { Beobachtel: } \\
33^{0} 20^{\prime}\end{array}$} \\
\hline$(001):(705)$ & $54 \quad 4$ & 54 & 8 \\
\hline$(001):(509)$ & $28 \quad 41 \frac{1}{2}$ & 28 & 31 \\
\hline$(001):(302)$ & 5555 & 55 & $52 \frac{1}{2}$ \\
\hline$(001):(10.0 .7)$ & 54.37 & 54 & 38 \\
\hline$(001):(403)$ & 5244 & $\mathbf{5 2}$ & 423 \\
\hline$(001):(503)$ & $58 \quad 40$ & 58 & 38 \\
\hline$(001):(313)$ & $\begin{array}{ll}46 & 6\end{array}$ & 46 & 1 \\
\hline$(001):(1 T 3)$ & 24542 & 24 & 45 \\
\hline$(001):(771)$ & $848 \frac{3}{4}$ & 84 & 13 \\
\hline$(111):(771)$ & 2953 & 29 & $55 \frac{3}{4}$ \\
\hline$(111):(101)$ & $35 \quad 3 \frac{3}{4}$ & 35 & $4 \frac{1}{2}$ \\
\hline$(111):(201)$ & $39 \quad 5 \frac{1}{2}$ & 39 & $5 \frac{1}{2}$ \\
\hline$(111):(203)$ & $3626 \frac{1}{2}$ & 36 & 25 \\
\hline$(111):(705)$ & $36 \quad 9 \frac{1}{2}$ & 36 & 10 \\
\hline$(111):(302)$ & $3637 \frac{1}{2}$ & 36 & 35 \\
\hline$(111):(1 T 1)$ & $70 \quad 7 \frac{1}{2}$ & 70 & 6 \\
\hline$(111):(11 T)$ & 7120 & 71 & $17 \frac{1}{2}$ \\
\hline$(111):(113)$ & $29 \quad 25 \frac{1}{2}$ & 29 & $26 \frac{3}{4}$ \\
\hline$(111):(313)$ & 2154 & 21 & $58 \frac{1}{2}$ \\
\hline$(101):(10 \pi)$ & 9051 & 90 & 51 \\
\hline$(101):(T 01)$ & $\begin{array}{ll}89 & 9\end{array}$ & 89 & $8 \frac{1}{2}$ \\
\hline$(101):(023)$ & 5328 & 53 & $25 \frac{3}{4}$ \\
\hline$(101):(705)$ & $929 \frac{1}{2}$ & 9 & 21 \\
\hline$(101):(313)$ & 1310 & 13 & 6 \\
\hline$(110):(111)$ & $35 \quad 40$ & 35 & 39 \\
\hline$(110):(101)$ & 65154 & 65 & 14 \\
\hline$(110):(771)$ & $5 \quad 46 \frac{1}{4}$ & $\boldsymbol{5}$ & 47 \\
\hline$(201):(20 \pi)$ & $53 \quad 49$ & $\mathbf{5 3}$ & 45 \\
\hline$(201):(021)$ & $78 \quad 11$ & 78 & 11 \\
\hline$(203):(023)$ & $45 \quad 41$ & 45 & 40 \\
\hline$(203):(\overline{2} 03)$ & $66 \quad 37$ & 66 & 32 \\
\hline$(771):(113)$ & $59 \quad 14 \frac{1}{4}$ & 59 & 18 \\
\hline
\end{tabular}

Die Krystalle selbst sind ausserordentlich schön. Ihre Flächen sind nebst der charakteristisch gelben Farbe meistens glänzend und nur selten matt, was oft röthliche oder violette Anlauffarben nach sich zieht.

Die einfachen Krystalle und die Zwillinge sind sehr verschiedenartig, können aber auf einige Haupttypen zurückgeführt werden.

Einfache Krystalle. Bei den ziemlich seltenen einfachen Krystallen habe ich vier Typen unterschieden. 
1) Am häufigsten ist jene pyramidale Gestalt zu finden, bei der die Pyramiden zweiter Art herrschen. Nanchmal tritt nur die von $x\{111\}$ und $x\{1 T 1\}$ abgestumpfte $e\{101\}$ für sich auf, oder es sind neben ihr auch $z\{201\}, g\{203\}, e\{001\}, m\{110\}$ und nicht selten auch $a\{100\}$ vorhanden. Die Pyramidenflächen sind hier alle glatt und glänzend, wie auch jene des negativen Sphenoids. Lin wenig gestreift sind hingegen die Flächen von $\{110\}$, gekörnt die von $\{001\}$, während das positive Sphenoid $\%\{111\}$ horizontal gerieft ist und deshalb undeutliche leflexe liefert.

2) Seltener ist schon jener Typus, dessen herrschende Formen die Grundsphenoide sind, in ziemlichem Gleichgewicht mit $\{101\},\{201\}$ und \{203\}. Die positiven Sphenoide sind hier weniger gerieft.

3) Der dritte, prismatische Typus ist an Formen auch nicht reich, so dass ich ausser $x\{111\}, x\{111\},\{101\},\{201\},\{203\},\{110\},\{100\}$ und $\{001\}$ keine anderen beobachten konnte.

Wie es dic Fig. 3 zeigt, sind an den Krystallen die stark gerieften Prismenflächen $\{110\}$ und die glïnzende Endfläche überwiegend. Als einfiacher Krystall kam dieser T'ypus zwar nur einmal vor, wiederholt aber in Zwillingen.

4) Als vierter Typus kann das positive Sphenoid $\approx\{111\}$ erwähnt werden, von dem ich nur ein Exemplar fand, mit stark gerieften, verzerrten Flïchen und von $x\{1 T 1\}$ abgestumpften Ecken.

Zwillingskrystalle. Am untersuchten Material waren zwei Zwillingsgesetze vertreten.

1) Bei dem ersten ist eine Fläche des Grundsphenoids die Zwillingsebene; sehr charakteristisch ist hier der von $\{111\}$ und $\{111\}$ gebildete Winkel $37^{\circ} 20^{\prime}$; er ist gewöhnlich schr gut erkennbar und darum zur Orientirung am geeignetsten. Während $x\{111\}$ geriefl erscheint, ist seine andere Fläche $*\{111\}$ immer glïnzend, da sich in der Kante ungleichnamige Sphenoide treffen.

Die grosse Mehrzahl der unlersuchten Krystalle gehorcht diesem Gesetze, zwei-, drei- bis vierfache oder polysynthetische Zwillinge bildend.

Die zweifachen Zwillinge kïnnen an dem schon erwähnten Winkel $\left(37^{0} 20^{\prime}\right)$ leicht erkannt werden. Sie sind thcils aus prismatischen, theils aus sphenoidalen Individuen zusammengesetzt. Im ersten Falle entsprechen sie der Fig. 1 und weichen davon nur insofern ab, dass entwetler das relative Grössenverhültniss von $\{001\}$ und $\{110\}$ schwankt, oder einer der beiden Componenten sehr reducirl, als ziemlich dünne Platte erscheint.

Dic Zwillinge des sphenoidalen Typus sind schon formenreicher; so fand ich an einem cine ganze lheihe der Pyramiden zweiter Art, nämlich: $\approx\{201\}, h\{302\}, \delta\{10.0 .7\}, \varepsilon\{705\}, \pi\{403\}, e\{101\}$ und $g\{203\}$, nebst. den anderen, gewohnten liormen. 
Die drei-, vier- und fünffachen Zwillinge dieses Geselzes bilden die Mehrzahl des bearbeiteten Materials.

Von den dreifachen Zwillingen ist besonders jener bemerkenswerth, den ich in Fig. 5 naturgetreu darzustellen versuchte. Der Winkel $x\{111\}$, $x\{111\}=37^{\circ} 20^{\prime}$ wiederholt sich hier noch einmal; daraus wird es klar, dass zwei Individuen in Form eines schrägen Kreuzes einander vollständig durchdringen, während sich an das eine in derselben Zone noch ein drittes - ebenfalls nach dieser Regel - anfügt. Und da die Prismenfläche $m\{1 / 0\}$ dem ganzen Krystallcomplexe gemeinsam ist, so entsteht eine roseltenähnliche Figur. An diesem Krystalle habe ich übrigens auch die neue Form $\eta\{771\}$ gefunden.

Fig. 6 stellt einen Drilling dar, bei dem die Individuen eine andere Situation haben. Hier kommen nämlich zwei zu einander senkrechte Zonen in Betracht; in der einen ist das negative, in der anderen das positive Sphenoid die Zwillingsebene, während im vorigen Falle beide derselben Zone angehörten. An diesem Exemplare war auch $\{509\}$ mit drei ziemlich guten Flächen und ferner $*\{113\}$ nebst $*\{113\}$ vorhanden.

2) Das zweite $Z_{\text {willingsgesetz, welches an diesem Kupferkies vertreten }}$ war, wurde zuerst von Mohs erwähnt $\left.{ }^{1}\right)$. Zwillingsebene ist hier eine Fläche der Pyramide $e\{101\}$.

Für die Krystalle, welche derart gebildet sind, habe ich nur eine Bemerkung. Baumhauer ${ }^{2}$ ) erwähnt in der Beschreibung der Burgholdinghausener Kupferkiese, dass dieselben der Regel nie vollständig gehorchen, sondern ein Bestreben aufweisen, ihre Endflächen in gegenseitig senkrechte Lage zu bringen. Das ist bei den vorliegenden Krystallen nicht der Fall, da die beobachteten und berechneten Winkelwerthe, wie im Folgenden ersichtlich, sehr gut übereinstimmen.

\begin{tabular}{|c|c|c|}
\hline$(001):(001)$ & $\begin{array}{l}\text { Bereclinet: } \\
=90^{\circ} 51^{\prime}\end{array}$ & $\begin{array}{r}\text { Beobachitel } \\
90^{\circ} 491\end{array}$ \\
\hline$(101):(101)$ & 142 & $14.1 \%$ \\
\hline$(111):(111)$ & 1233 & 123 \\
\hline$(302):(302)$ & 2059 & - \\
\hline$(203):(\underline{203})$ & 2358 & $21 \quad 21$ \\
\hline$(101):(001)$ & $\begin{array}{ll}46 & 161 .\end{array}$ & $46 \quad 121$ \\
\hline
\end{tabular}

Ilọ̈chst charakteristisch sind bei diesem Kupferkiese jene vier- und sechsfachen Zwillinge, an denen nach dem letzterwähnten Gesetze zwei Individuen verwachsen, die wieder für sich mit einem oder zwei anderen im Sinne des ersten Gesetzes verbunden sind. Da die entsprechenden Theile meist gleich gross entwickelt sind, bekommt der ganze Krystallstock ein ziemlich symmetrisches Aussehen. 
Der Unlerschied zwischen den positiven und negaliven Sphenoidflächen ist hier auch so ausgeprägt, wie beim vorigen Typus, wodurch eine weitere Analyse der Krystalle auf keinerlei Schwierigkeiten stösst.

Diese combinirte Zwillingsbildung ist übrigens eine höchst charakterislische Eigenschaft des Pulacayoer Kupferkieses, die ich von anderen Fundorten noch nicht erwähnt fand.

Ausser diesen eigentlich krystallographischen Beobachtungen kann ich noch meine Experimente mittheilen, mit denen ich die sichere Unterscheidung der ungleichnamigen Sphenoide zu erreichen versuchte, da uns die Literatur in dieser Hinsicht keine festen Gesichtspunkte bietet. Sade-. beck ${ }^{1}$ ) und Schimper ${ }^{2}$ ) glaubten zwar an dem glänzenden oder gerieften Aeusseren der Sphenoidflächen den negativen resp. positiven Charakter zu erkennen; manchmal erreichten sie dadurch ihr Ziel, oft waren aber $*\{111\}$ und $x\{1 T 1\}$ in physikalischer Hinsicht vollkommen gleich, wodurch eine Orientirung unmöglich wurde. So beschreibt Sadebeck selbst ${ }^{3}$ ) (1886) vom Stahlberg bei Müsen solche Krystalle in einer Weise, welche seiner späteren Angabe $(1868,1878)$ widerspricht.

Die Resultate meiner Aetzversuche sind folgende: In sehr verdünnter Salpetersäure 15-30 Minuten lang gekocht erlitten die Krystalle nur insofern eine Aenderung, dass ihre Flächen, mit Ausnahme der glänzenden, "negativen "Sphenoide, violette Anlauffarben bekamen. Beim Gebrauche einer stärkeren Lösung wurden die Flächen schon bemerkbar angegriffen, das erwähnte Sphenoid zeigte aber nur jene Farben, wie die übrigen vorher. Das Verhalten der Krystalle verrith also schon, dass $\approx\left\{1 T_{1}\right\}$ entschieden widerstandsfähiger ist, als die anderen Flächen.

Anwendung von Schwefelsäure hatte denselben Erfolg; doch Aetzfiguren gelang es mir nur dann zu erzeugen, wenn ich concentrirte und erhitzte Säure verwendete. Am schünsten wurden die vertieften Figuren, wenn ich die Krystalle an dünnem Platindraht befestigt auf $11-1 \frac{1}{2}$ Min. in die schon voraus zum Sieden erhitzte Säure tauchte. Unterdessen wurden zwar die Flächen sehr corrodirt, $\star\{1 T 1\}$ zejgte aber zierliche Aetzfiguren, welche die sphenoidale Symmetrie des Kupferkieses bewiesen.

Der Kupferkies wird von Quarz, Fahlerz, Zinkblende und kleinen Pyrargyritkryställchen begleitet.

Die Mischung derben Kupferkieses mit chenfalls compactem oder krystallinischem Pyrit bedeckt eine Schicht drusigen Quarzes, aus welcher sich die schönen Kupferkieskrystalle erheben. Auf diese Mineralien folgt das Fahlerz und die Blende. An manchem Exemplare sind besonders die Tetra-

1) Zeitschr. d. d. geol. Ges. 1868, 1878.

2) Groth, Miner. Samml. Strassburg 1878.

3) Zeitschr. d. d. geol. Ges. 1866. 
¿̈drite, aber auch die Sphalerite mit einer jüngeren Generation des Kupferkieses bestreut. Bei einem Stücke fanden sich auch winzige Pyrargyritkryställchen auf den Kupferkies gelagert, die eine sehr interessante Art der regelmässigen Verwachsung aufweisen.

Der krystallisirte Eisenkies erscheint in 0,5-2 mm grossen, röthlichen, manchmal angelaufenen und charakteristisch gerieften Pentagondodekaëdern, nicht selten mit dem Oktaëder oder mit Spuren des Ilexaëders.

An den gut ausgebildeten Krystallen des Fahlerzes (ca. $0,5-1,5 \mathrm{~cm}$ ) beschrieb G. vom Rath in der schon erwähnten Abhandlung die Formen

$x\{211\}, x\{332\}, x\{111\},\{110\}, x\{774\}$ und Zwillinge nach $\{111\}$.

Die Sphalerite sind nur selten zur Messung geeignet; nur $\{110\}$ und \{311\} konnten an ihnen nachgewiesen werden.

Die kleinen, mit freiem Auge kaum bemerkbaren Krystalle der Antimonsilberblende bcherrscht $a\{11 \overline{2} 0\}$ und $r\{10 \bar{T} 1\}$, während das Skalenoëder $v\{12 \overline{3} 5\}$ und einige undeutliche kleine lilächen nur eine untergeordnete Rolle spielen.

Die Pyrargyrite sind besonders auf den Flächen der Pyramide Il. Art $c\{101\}$ gut entwickelt, und zwar in der Weise, dass ihre Hauptaxen einer Polkante dieser Form, z. B. $[(101):(011)]$ parallel sind. (Siehe Fig. 8 und 9.) Daraus folgt nun, dass sämmtliche Prismenflächen des Pyrargyrits mit $e(101), e^{\prime}(011)$ und natürlich auch $m(1 \bar{T} 0)$ des Kupferkieses tautozonal sind. In dieser Zone war aber:

\begin{tabular}{|c|c|}
\hline Berechnet: & Beobachtei: \\
\hline$a: e^{\prime}=0^{0} 15 y^{\prime}$ & $0^{0} 1 \ddot{a}^{\prime}-20^{\prime}$ \\
\hline$e^{\prime}: e=59301$ & 5930 \\
\hline$c: a^{\prime}=0$ & $0 \quad 16-20^{\prime}$ \\
\hline $\begin{array}{l}a^{\prime}: a^{\prime \prime}=60 \\
e: a^{\prime \prime}=60 \quad 15 \%\end{array}$ & 60 circa, woraus \\
\hline
\end{tabular}

Andererseits ist auch $e(101): m(10)=600151$, weshalb $a^{\prime \prime}(11 \overline{2} 0)$ mit $m(1 T 0)$ zugleich einspiegelt. Da nun $m$ einer Symmetrieebene des Kupferkieses, $a$ " hingegen einer des Pyrargyrites parallel ist, kann' das Gesetz so ausgesprochen werden: Die $z$ wei Minerale sind so mit einander vcrwachsen, dass je eine ihrer Symmetrieebenen zusammenfällt und die Hauptaxen der hexagonalen Prismen einer Polkante der Form $e\{101\}$ parallel sind. Da der Kupferkies zwei. zu einander senkrechte Symmetrieebenen besitzt, sind auf jeder seiner Flächen zwei Systeme der Pyrargyrite möglich.

In der Literatur fand ich für Kupferkies schon mehrere regelmässige 
Verwachsungen erwähnt. So beobachteten Pelikan, Souheur, Lüdeckel) Bleiglanz, Haidinger, II ausmann, Sadebeck, Frenzel und Becke²) Zinkblende, Mügge ${ }^{3}$ ) Kobaltglanz, Wa ckernagel, Breithaupt, Sadcbeck, Zincken und Rammelsberg $\left.{ }^{4}\right)$ Fahlerz, ferner Haidinger ${ }^{5}$ ) Silberglanz, Frenzel $\left.{ }^{0}\right)$ Polylsasit und Bournonit mit ähnlicher Regelmässigkeit aufgewachsen.

Von der Verwachsung des Kupferkieses mit Pyrargyrit wird aber weder in M. F. Wallerant's noch in Mügge's zusammenfassender $\Lambda \mathrm{b}$ handlung cine Erwähnung gemacht, wesshalb die beschriebene Erscheinung als neue gelten kann.

Endlich sei es mir erlaubt, meinem hochgeehrten Lehrer, Herrn Prof. Josef Krenner, den innigsten Dank auszusprechen sowohl für das prachtvolle Material, wie für scine werthen Rathschläge, mit denen er mich stets unterstützte.

Mineralogisch-petrographisches Institut der Universilït in Budapest.

1) Pelikan, Tscherm. min. u. petr. Mitth. 1892. - Souheur, diese Zeitschr. 1894. - Lü de cke, Miner. des Harzes 1896.

2) Haidinger, Handbuch d. best. Miner. 184. - Hausmann, Abh. Götting. Ges. 1856. - Sadebeck, Zeitschr. d. d. geol. Ges. 1872. - Frenzel, Min. Lex. v. Sachsen 1874. - Becke, Tscherm. min. u. pelr. Milll. 1883.

3) M ügge, Tscherm. min. u. petr. Mith. 1883 .

4) Wackernage l, Kaslner's Arch. f. ges. Nat. 1825. - B jeith a upt, Berg.u. Hütt. Zeitg. 1861. - Sadebeck, Zeitschr. d. d. geol. Ges. 1872. - Zincken und Rammels berg, Pogg. Ann. 1849.

5) Il a id inger, Ilandb. d. best. Min. 1845.

6) Frenzel, Nin. Lex. v. Sachsen 1866, 1877. 